\title{
Effect of Fullerene Encapsulation on Radial Vibrational Breathing-Mode Frequencies of Single-Wall Carbon Nanotubes
}

\author{
Soon-Kil Joung, ${ }^{1}$ Toshiya Okazaki, ${ }^{1,2, *}$ Naoki Kishi, ${ }^{1, \dagger}$ Susumu Okada ${ }^{3}$ Shunji Bandow, ${ }^{4}$ and Sumio Iijima ${ }^{1,4}$ \\ ${ }^{1}$ Research Center for Advanced Carbon Materials, National Institute of Advanced Industrial Science and Technology (AIST), \\ Tsukuba 305-8565, Japan \\ ${ }^{2}$ PRESTO, Japan Science and Technology Agency, 4-1-8 Honcho, Kawaguchi 332-0012, Japan \\ ${ }^{3}$ Institute of Physics and Center for Computational Sciences, University of Tsukuba, 1-1-1 Tennodai, Tsukuba 305-8577, Japan, \\ and CREST, Japan Science and Technology Agency, 4-1-8 Honcho, Kawaguchi 332-0012, Japan \\ ${ }^{4}$ Department of Materials Science and Engineering, Meijo University, 1-501 Shiogamaguchi, Tenpaku-ku, Nagoya 468-8502, Japan
}

(Received 13 March 2009; published 10 July 2009)

\begin{abstract}
We investigate the effects of $\mathrm{C}_{60}$ fullerene encapsulation on the radial breathing mode (RBM) of semiconducting single-wall carbon nanotubes (SWCNTs) under tunable laser excitations. The changes in the RBM frequencies after $\mathrm{C}_{60}$ insertions show characteristic behavior; higher frequency shifts are observed in the case of smaller diameter tubes $\left(d_{t} \leqslant 1.32 \mathrm{~nm}\right)$ and lower frequency shifts, in the case of larger diameter tubes $\left(d_{t} \geqslant 1.32 \mathrm{~nm}\right)$. The observed frequency shifts are satisfactorily explained by the diameter-dependent interaction between the encapsulated $\mathrm{C}_{60}$ and the host SWCNTs.
\end{abstract}

DOI: 10.1103/PhysRevLett.103.027403

PACS numbers: 78.67.Ch, 73.22.-f, 78.30.Na

Single-wall carbon nanotubes (SWCNTs) exhibit unique molecular and electronic structures, which are associated with their tubular morphologies. One such interesting property of SWCNTs is that the space within the nanotubes has the same size of the organic molecule so that the effective interaction between the guest molecules and the host SWCNTs can be predicted. In fact, there exists a so-called nearly-free-electron (NFE) state inside the SWCNTs, and this state is distributed away from the carbon sites [1]. When guest molecules are introduced within the SWCNTs, the electronic states of the guest substantially interact with the NFE states of the nanotubes, giving rise to a novel electronic structure of the SWCNTs [2].

For monitoring the host-guest interaction in SWCNT systems, Raman spectroscopy is one of the most suitable and efficient techniques [3]. The radial breathing mode (RBM) phonon of SWCNTs is particularly useful, since its frequency is directly related to the inverse tube diameter $\left(1 / d_{t}\right)$ and is sensitive to molecular encapsulation. Theoretical calculations have predicted that the frequency shift of RBM phonon is observed when $\mathrm{C}_{60}$ molecule is encapsulated in a metallic armchair SWCNTs depending on the tube diameter [4]. This is illustrated by the fact that a $(10,10)$ nanotube exhibited an upshift in the RBM frequency on encapsulation, whereas the $(11,11)$ and $(12,12)$ SWCNTs with greater diameters exhibited downshifts in RBM frequencies [4].

Because of the resonance effect, the Raman intensity of SWCNTs drastically changes with the tube chirality and the excitation wavelength. Although the shift in the RBM frequencies on chemical and electrochemical doping has been frequently reported, the observed shifts seem to reflect the change in resonance enhancement in a mixture of SWCNTs rather than an actual shift in the RBM mode of a particular $(n, m)$ tube [5]. Therefore, for comprehensive characterizations, it is necessary to measure the Raman spectra by using several laser excitation lines.

In this Letter, we report the effect of $\mathrm{C}_{60}$ fullerene encapsulation on the RBM frequencies of SWCNTs by carrying out Raman spectroscopy with laser excitations tuned from 1.19 to $1.65 \mathrm{eV}$; these excitations correspond to the second optical transitions $\left(E^{S}{ }_{22}\right)$ in the semiconducting SWCNTs. To study the abovementioned effects, we constructed the two-dimensional (2D) contour maps of the resonance Raman intensities using $\mathrm{C}_{60}$ nanopeapods and a SWCNTs control sample in micellar solutions. The observed RBM peaks before and after the encapsulation are studied to elucidate the cause of the frequency shifts in RBM phonon.

Figure 1 shows the resonance Raman spectra of the SWCNT control sample and $\mathrm{C}_{60}$ nanopeapods in the RBM frequency region under different excitation energies [6]. In the spectra of the SWCNT control sample [Fig. 1(a)], prominent RBM phonon peaks are observed at around 199 and $185 \mathrm{~cm}^{-1}$ under the excitations of $\sim 1.33 \mathrm{eV}$ and $\sim 1.25 \mathrm{eV}$, respectively. Strong excitation-energy dependence is a natural consequence of the chirality distribution of the electronic transitions in the SWCNTs.

In the case of the $\mathrm{C}_{60}$ nanopeapods [Fig. 1(b)], the spectral shapes and peak positions are relatively complicated, and additional peaks are observed at RBM phonon frequencies other than those mentioned above. Under relatively high excitation energy of $1.33 \mathrm{eV}$, a strong RBM phonon peak was observed at $\sim 199 \mathrm{~cm}^{-1}$, as in the case of the SWCNT control sample. As the excitation energy decreases, the intensity of this peak decreases and another peak appears at $\sim 193 \mathrm{~cm}^{-1}$ at the excitation of $\sim 1.28 \mathrm{eV}$. Further decrease in energy excitation to $\sim 1.24 \mathrm{eV}$ results 

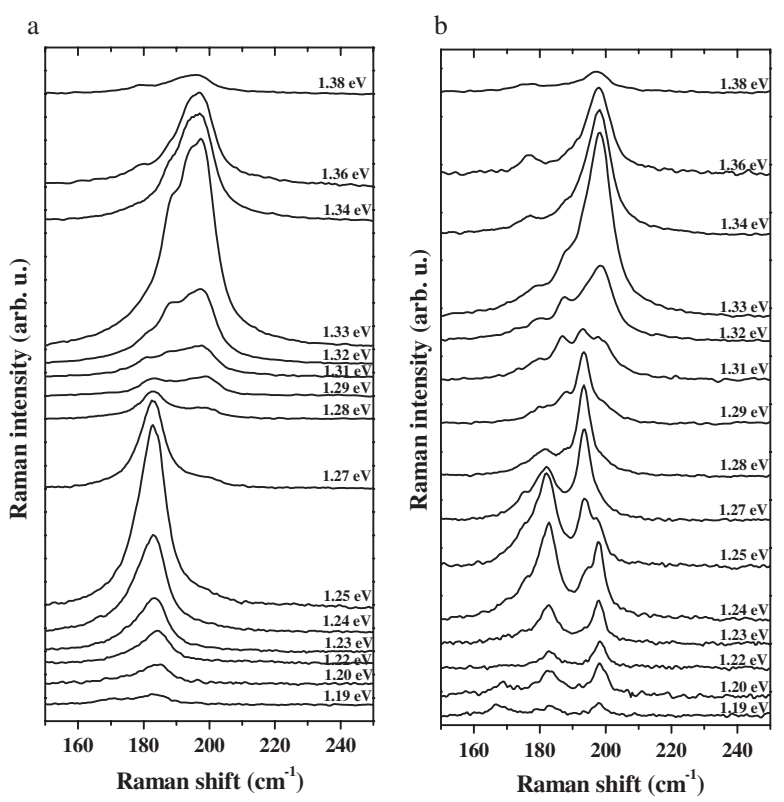

FIG. 1. Resonance Raman spectra in RBM region of (A) SWCNTs control samples in $\mathrm{SDBS}_{-} \mathrm{D}_{2} \mathrm{O}$ solution and (B) $\mathrm{C}_{60}$ nanopeapods in $\mathrm{SDBS}-\mathrm{D}_{2} \mathrm{O}$ solution under different excitation energies.

in the appearance of a strong RBM phonon peak at $\sim 198 \mathrm{~cm}^{-1}$ in addition to an RBM peak at $\sim 180 \mathrm{~cm}^{-1}$ which is also observed in the case of the control sample.

In order to assign these RBM phonon peaks to the respective $(n, m)$ tubes, we constructed a $2 \mathrm{D}$ contour map from the resonance Raman spectra of the RBM phonon region from 1.19 to $1.65 \mathrm{eV}$. Figure 2(a) shows the 2D RBM intensity map of the SWCNT control sample in the SDBS- $\mathrm{D}_{2} \mathrm{O}$ solution. The white circles in Fig. 2 indicate the RBM phonon peak positions in the individual $(n, m)$ tubes obtained by the line-shape fitting. Typical $2 n+m$ family patterns [7,8] are clearly observed in Fig. 2(a). The RBM phonon peaks appearing between $175-185 \mathrm{~cm}^{-1}$ under the excitation wavelength region of $1.23-1.27 \mathrm{eV}$ belong to a single $2 n+m$ branch. Another branch is clearly delineated in the frequency range of $181-199 \mathrm{~cm}^{-1}$ under excitation wavelengths from 1.31 to $1.36 \mathrm{eV}$. On the basis of the photoluminescence excitation (PLE) and Raman results reported in a previous study [9], the $2 n+m$ branches observed in our results can be assigned to the $2 n+m=28,29,31,32$, and 34 families, as shown in Fig. 2(a).

The 2D RBM intensity map of $\mathrm{C}_{60}$ nanopeapods is shown in Fig. 2(b). The general Raman features are very similar to those of the SWCNT control sample [Fig. 2(a)]. For example, the RBM phonon peaks assignable to SWCNTs with $2 n+m=28,29,31$, and 32 families are observed in Fig. 2(b) at the same frequencies as those in the case of SWCNT control sample.

Although the general Raman features of the $\mathrm{C}_{60}$ nanopeapods and SWCNT control sample are comparable [Figs. 2(a) and 2(b)], there are some characteristic differ-
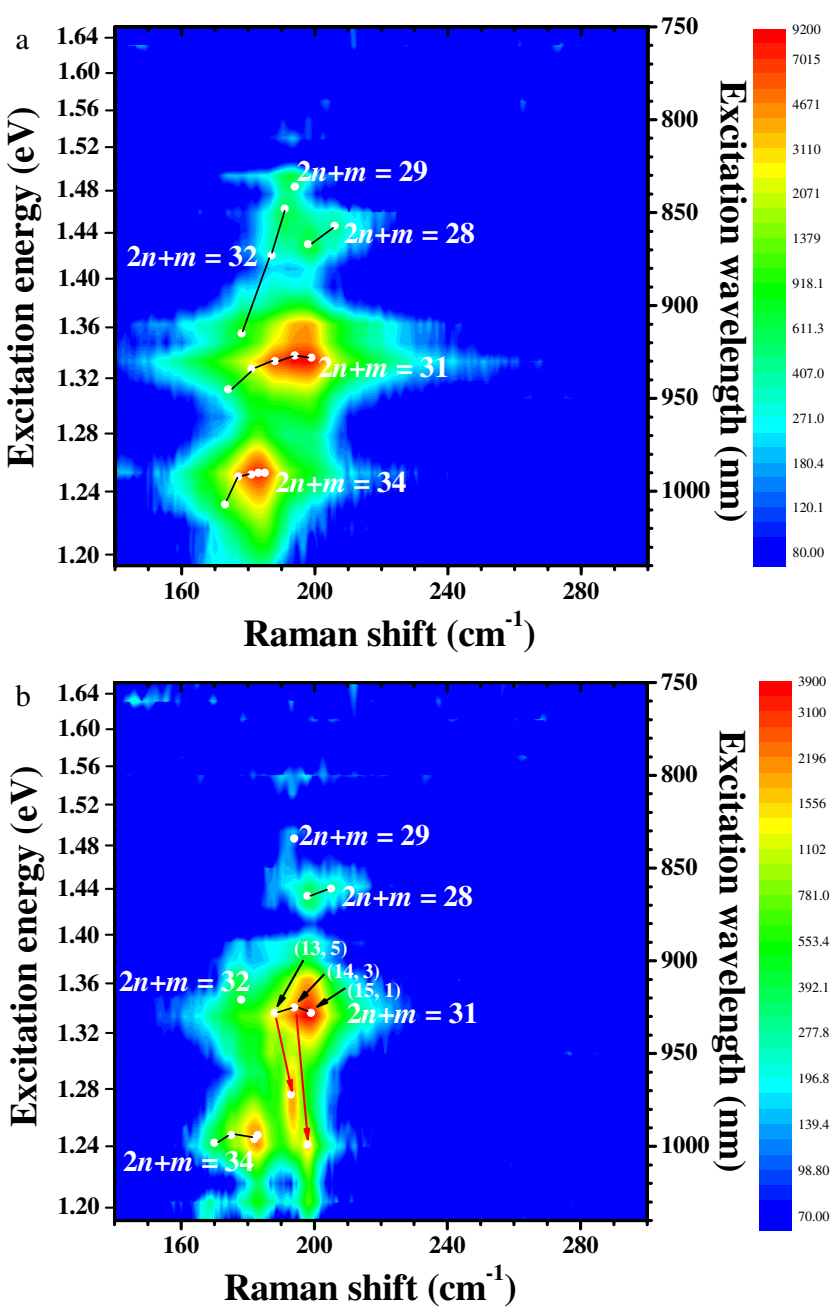

FIG. 2 (color online). 2D Raman intensity maps of RBM phonon regions of (a) SWCNTs control samples and (b) $\mathrm{C}_{60}$ nanopeapods in SDBS- $\mathrm{D}_{2} \mathrm{O}$ solution. Color patterns follow a $\log$ scale to clearly show the low intensity RBM peaks.

ences between the two spectra. The RBM peaks observed between $170-183 \mathrm{~cm}^{-1}$ under the excitation energies of $1.23-1.27 \mathrm{eV}$ can be assigned to the $2 n+m=34$ family because these peaks show the typical "hooked" family pattern [7,8]. However, the RBM frequencies of these peaks show lower shifts of approximately $1-7 \mathrm{~cm}^{-1}$ in the case of the $\mathrm{C}_{60}$ nanopeapods as compared to those in the case of SWCNTs ( see Table 1 in [6]).

Furthermore, in addition to the peaks corresponding to the $2 n+m=28,29,31,32$, and 34 branches, two new peaks are observed at 193 and $198 \mathrm{~cm}^{-1}$ under the excitation energies of $\sim 1.28 \mathrm{eV}$ and $\sim 1.24 \mathrm{eV}$, respectively, in the case of the $\mathrm{C}_{60}$ nanopeapods. Previous PLE studies have revealed that the optical transition energies of SWCNTs change after $\mathrm{C}_{60}$ encapsulation [10]. In particular, the optical transition energies of smaller diameter tubes such as those with $(13,5)$ and $(14,3)$ structures are strongly affected by $\mathrm{C}_{60}$ insertion. The optical transition energies of $(13,5)$ and $(14,3)$ tubes change from 1.333 to $1.268 \mathrm{eV}$, 
and 1.335 to $1.223 \mathrm{eV}$, respectively [10]. The energies 1.268 and $1.223 \mathrm{eV}$ are comparable to the resonance energies of the two new RBM peaks $(\sim 1.25$ and $\sim 1.22 \mathrm{eV})$. Further, the RBM frequencies corresponding to the $(13,5)$ and $(14,3)$ tubes (i.e., 188 and $194 \mathrm{~cm}^{-1}$, respectively) are not significantly different from those of the two new RBM peaks. Therefore, we conclude that these new RBM peaks observed at 193 and $198 \mathrm{~cm}^{-1}$ are attributed to $(13,5)$ and $(14,3)$ nanopeapods, respectively. These assignments are further verified by the fact that the RBM intensity ratios of these nanotubes and the $(15,1)$ tube decrease $\sim 50 \%$ after $\mathrm{C}_{60}$ encapsulations because $(15,1)$ tubes have too small diameter to encapsulate $C_{60}$ [10] [Fig. 2(b)]. The whole RBM peaks observed here can be reasonably assigned to the $\mathrm{C}_{60}$ nanopeapods and the original SWCNTs. It was unnecessary to assume the additional RBM peaks originated from another species.

It is worth noting that the change in the RBM phonon frequency on encapsulation is observed only in the case of SWCNTs with $d_{t}>1.25 \mathrm{~nm}$. The diameter of $1.25 \mathrm{~nm}$ exactly corresponds to the smallest limit for $\mathrm{C}_{60}$ encapsulation, as determined by the PLE studies [10]. This strongly suggests that $\mathrm{C}_{60}$ is preferentially encapsulated into SWCNTs with $d_{t}>1.25 \mathrm{~nm}$ and that this filling of SWCNTs results in the RBM frequency shifts of those SWCNTs. The observed RBM phonon frequencies of SWCNTs with $d_{t}>1.25 \mathrm{~nm}\left(\omega^{\text {SWCNTs }}\right)$ and those of the corresponding $\mathrm{C}_{60}$ nanopeapods ( $\left.\omega^{\text {peapods }}\right)$ are summarized in Table 1 of [6]. All the SWCNTs listed in Table 1 are classified as type I because the RBM phonon intensity for second interband transition is much higher than those of type II tubes (type I $[\bmod (2 n+m, 3)=1]$ and type II $[\bmod (2 n+m, 3)=2])[6,11-13]$.

To investigate the fullerene encapsulation effect on the RBM frequency of SWCNTs in further detail, the frequency difference before and after insertion $(\Delta \omega=$ $\left.\omega^{\text {peapods }}-\omega^{\text {SWCNTs }}\right)$ is plotted as a function of the tube diameter [Fig. 3(a), solid circles]. In the case of tubes with smaller diameters, such as the $(14,3)$ and $(13,5)$ tubes, high-frequency shifts were observed. With increasing tube diameter, the $\Delta \omega$ value monotonically decreases and becomes negative. The calculated $\Delta \omega$ values for several zigzag $(n, 0)$ and armchair $(n, n)$ tubes are also plotted in Fig. 3(a) (open circles). From this figure, it is clearly seen that the diameter dependence of the RBM frequency shifts of the SWCNTs is reproduced in our theoretical calculations.

The upward shifts observed in the case of smaller diameter tubes can be explained by the fact that in these tubes, there is steric hindrance provided by the encapsulated $\mathrm{C}_{60}$ for RBM motion of SWCNTs [4]. The insertion of an oversized $\mathrm{C}_{60}$ molecule directly suppresses the radial vibration motion of SWCNTs. The local strain on the SWCNTs after $\mathrm{C}_{60}$ encapsulation may disappear at $\sim 1.32 \mathrm{~nm}$ because the frequency shift at this diameter changes the sign [Fig. 3(a)]. Interestingly, this value is also close to the nearest-neighbor distance between the
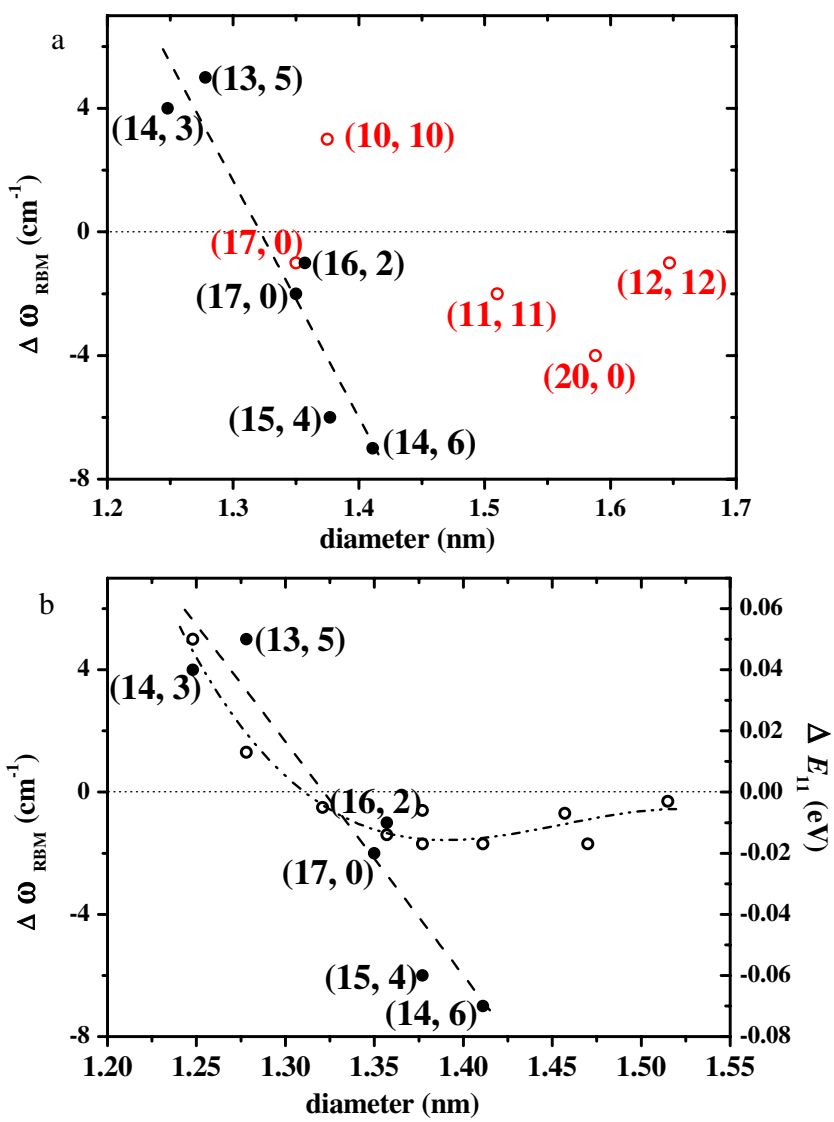

FIG. 3 (color online). (a) Experimentally obtained $\Delta \omega_{\mathrm{RBM}}$ (solid circles) and calculated $\Delta \omega_{\mathrm{RBM}}$ (open circles) as a function of tube diameter. (b) Experimentally obtained $\Delta \omega_{\mathrm{RBM}}$ (solid circles) and $\Delta E_{11}$ of type I tubes (open circles) as a function of tube diameter.

$\mathrm{C}_{60}$ molecule and the SWCNT, which can be obtained by a simple calculation. The intermolecular distance between $\mathrm{C}_{60}$ molecules present inside the SWCNTs was found to be $0.97 \mathrm{~nm}$ by electron diffraction methods [14]. It is well known that the interlayer distance between two graphenes in graphite is $0.34 \mathrm{~nm}$ [15]. Hence, the smallest distance from the center of the $\mathrm{C}_{60}$ molecule to the tube wall can be calculated to be $0.66[=(0.97+0.34) / 2] \mathrm{nm}$, which suggests that a tube with diameter of more than $1.31(=0.97+$ $0.34) \mathrm{nm}$ is required so that a $\mathrm{C}_{60}$ molecule can enter the tube without friction. Excellent agreement about the threshold diameter strongly suggests that the RBM phonon shifts observed in the case of $(14,3)$ and $(13,5)$ tubes are induced by the repulsive interaction between SWCNTs and $\mathrm{C}_{60}$ arising from the limited spacing between them.

On the other hand, the downshifts observed in the case of larger diameter tubes can be explained by the hybridization between the electronic states of SWCNTs and $\mathrm{C}_{60}$ [4]. In tubes with such large diameters, the attractive interaction between SWCNTs and $\mathrm{C}_{60}$ was predicted by theoretical calculations $[4,16]$. This attractive interaction results in the effective coupling of the $\pi$ states of SWCNTs and $\mathrm{C}_{60}$ and the subsequent expansion of the $\pi$-electron cloud of 
SWCNTs and $\mathrm{C}_{60}$. This expansion induces charge redistribution, whereby the electrons are transferred from the $\pi$ orbital of both SWCNTs and $\mathrm{C}_{60}$ to the free space between them. In fact, the electron density in the vicinity of the tube wall is found to decrease $(\sim 0.05 e)$ [4]. This decrease in electron density should decrease the force constant of the $\mathrm{C}-\mathrm{C}$ bond, resulting in the downward shift of RBM phonon frequencies on $\mathrm{C}_{60}$ encapsulation.

Further, the diameter dependence of the RBM phonon frequency shifts is in good agreement with the previously reported PLE results $[10,17]$. We have previously demonstrated that the optical band gap of semiconducting SWCNTs is substantially modified on $\mathrm{C}_{60}$ encapsulation, depending on the tube diameter. The difference between the first transition energies before and after $\mathrm{C}_{60}$ encapsulation $\left(\Delta E_{11}=E_{11}\right.$ peapods $\left.-E_{11}^{\text {SWCNTs }}\right)$ shows characteristic dependence on the tube diameter. Figure 3(b) shows the $\Delta E_{11}$ values of type I tubes as a function of the tube diameter (open circles) obtained in a previous study [17] along with the present RBM results ( $\Delta \omega$, solid circles). The diameter dependence of $\Delta E_{11}$ can be explained as follows $[10,17]$. The positive $\Delta E_{11}$ value in the case of smaller diameter tubes $\left(d_{t} \lesssim 1.32 \mathrm{~nm}\right)$ can be attributed to the strain-induced band-gap shift that results from the expansion of the tube diameter due to the encapsulated $\mathrm{C}_{60}[10,17]$. On the other hand, the negative $\Delta E_{11}$ values in the case of larger diameter tubes $\left(d_{t} \gtrsim 1.32 \mathrm{~nm}\right)$ can be attributed to the hybridization between the $\pi$ states of SWCNTs and those of $\mathrm{C}_{60}[10,17]$. From the figure, it is clearly observed that the diameter dependence of $\Delta E_{11}$ is comparable to that of $\Delta \omega$. In particular, the $\Delta E_{11}$ and $\Delta \omega$ values crossover the zero line at almost the same diameter $\left(d_{t}=1.32-1.33 \mathrm{~nm}\right)$. These results strongly suggest that the basic mechanism of $\mathrm{C}_{60}$ encapsulation on band-gap modification is the same as that on RBM frequency shifts as observed in the present study.

In this study, we also find the tendency for the signal intensity and the spectral linewidth of the RBM to decrease after $\mathrm{C}_{60}$ encapsulation. For example, the peak intensity and the FWHM of the RBM change $\sim 0.6$ and $\sim 0.8$-fold in average, respectively, for $(17,0),(16,2),(15,4)$ and $(14,6)$ tubes. However, at present, we cannot find a clear empirical regularity and a possible mechanism for these changes. These points will be well understood by using samples containing fewer types of tubes $[18,19]$.

In summary, the effects of $\mathrm{C}_{60}$ fullerene encapsulation on RBM phonon frequencies of SWCNTs were investigated by scanning a Raman spectra from 1.19 to $1.65 \mathrm{eV}$ using a tunable Ti:sapphire laser. By assigning the observed RBM phonon peaks to the appropriate $(n, m)$ SWCNTs, we elucidated the detailed mechanism of the frequency shifts. In the case of smaller diameter tubes such as $(13,5)$ and $(14,3)$ SWCNTs, the RBM phonon frequencies are upshifted after $\mathrm{C}_{60}$ encapsulation because of the steric hindrance provided by the $\mathrm{C}_{60}$ molecules for the vibrational motion of SWCNTs. In contrast, larger diameter tubes such as those with $(17,0),(16,2),(15,4)$, and $(14,6)$ structures show downshifts in RBM phonon frequency, which can be attributed to the hybridization of the electronic states of the SWCNTs and those of $\mathrm{C}_{60}$ on $\mathrm{C}_{60}$ encapsulation. The diameter dependence of the RBM frequency shifts is reproduced in the DFT calculations and is consistent with the diameter dependence of the band-gap modification reported in the previous PLE studies.

We thank Dr. T. Nakanishi (AIST) for his helpful discussions. We also thank Mr. C. Jung (Sungkyunkwan University) for helping us in our experimental work. Computations were performed at YITP Kyoto University, ISSP University of Tokyo, and RCCS Okazaki National Institute.

*Corresponding author.

toshi.okazaki@aist.go.jp

${ }^{\dagger}$ Present address: Graduate School of Engineering, Nagoya Institute of Technology, Nagoya 466-8555, Japan.

[1] S. Okada, A. Oshiyama, and S. Saito, Phys. Rev. B 62, 7634 (2000).

[2] T. Okazaki and H. Shinohara, in Applied Physics of Nanotubes: Fundamentals of Theory, Optics and Transport Devices, edited by S. V. Rotkin and S. Subramoney (Springer, Berlin, Germany 2005), Chap. 5, p. 133.

[3] R. Saito and H. Kataura, in Carbon Nanotubes: Synthesis, Structure, Properties, and Applications, edited by M.S. Dresselhaus, G. Dresselhaus, and Ph. Avouris) (Springer, Berlin, Germany, 2001), p. 213.

[4] S. Okada, Chem. Phys. Lett. 438, 59 (2007).

[5] L. Kavan and L. Dunsch, Chem. Phys. Chem. 8, 974 (2007) (and references therein).

[6] See EPAPS Document No. E-PRLTAO-103-038930 for experimental details and Table 1 in which the observed RBM frequencies of SWCNTs and $\mathrm{C}_{60}$ nanopeapods are listed. For more information on EPAPS, see http://www. aip.org/pubservs/epaps.html.

[7] H. Telg et al., Phys. Rev. Lett. 93, 177401 (2004).

[8] H. Telg et al., Phys. Rev. B 74, 115415 (2006).

[9] R. B. Weisman and S. M. Bachilo, Nano Lett. 3, 1235 (2003).

[10] T. Okazaki et al., J. Am. Chem. Soc. 130, 4122 (2008).

[11] M. Machón et al., Phys. Rev. B 71, 035416 (2005).

[12] V. N. Popov, L. Henrard, and P. Lambin, Nano Lett. 4, 1795 (2004).

[13] J. Jiang et al., Phys. Rev. B 71, 205420 (2005).

[14] K. Hirahara et al., Phys. Rev. B 64, 115420 (2001).

[15] H. Adam and R. Palser, Phys. Chem. Chem. Phys. 1, 4459 (1999).

[16] M. Otani, S. Okada, and A. Oshiyama, Phys. Rev. B 68, 125424 (2003).

[17] S. Okubo et al., J. Phys. Chem. C 113, 571 (2009).

[18] A. Nish et al., Nature Nanotech. 2, 640 (2007).

[19] F. Chen et al., Nano Lett. 7, 3013 (2007). 